\title{
A Comparative Study of Meta-heuristics Algorithms in evaluation of Economic Load Dispatch Problems in Power Generating Station with Matlab Codes
}

\author{
Osaremwinda OP*, Nwohu MN and Kolo JG \\ Department of Electrical and Electronics Engineering, Federal University of Technology, Minna, Nigeria
}

\begin{abstract}
This paper presents a comparative study of metaheuristics algorithms in evaluation of economic load dispatch problem in power generating station with MATLAB codes. In this paper, the formulation of the ELD problems using mathematical illustrations and MATLAB codes were presented. This consists of the ELD cost model, Model calculations, cost function and parse solution. It also presents the application of some metaheuristics algorithms (solution algorithms) such as ACSA and PSO in solving ELD problem with MATLAB codes. The ELD problem was modeling using Egbin thermal power station, Nigeria as our case study.
\end{abstract}

Keywords: Economic Load Dispatch (ELD); Ant Colony Search Algorithms (ACSA); Particle Swarm Optimization (PSO); MATLAB codes

\section{Introduction}

Economic Load Dispatch (ELD) is the reduction of the total cost of power generation (which includes fuel consumption and operational cost) of power generating plants while meeting the various loads demand and power losses in the power transmission system. The objective is to apportion the total load demand and total loss among the various generating units at the same time satisfying the system constraints with reduced generation costs. With Economic Load Dispatch, it is expected that the power utilities plan and forecast optimal load dispatch. Several considerations are made during energy scheduling, these amongst others are to find out the existing generating units, the distance between load centers and the generating units, identifying the operating limits of each generating units such as the ramp rate limits, maximum and minimum generation level, prohibited zones [1].

\section{Economic Load Dispatch}

Economic load Dispatch is to determine the real and reactive power scheduling in power system this is the minimization of the cost function of different generating units. For economic operation of the power system, the total load demand must be optimally shared among all the generating units with an objective to reduce the total generation cost [1]. This is also to find out the power outputs of all generating units in power system so that the total cost of generation of the system is minimized, while meeting the load demand, system equality and inequality constraints. The essential operation constraints are the power balance constraint, that is, the total generated power must be equivalent to the load demands plus the transmission losses on the power system, and the power limit constraints of the generating units [2-6]. The problem of economic operation of a power system is the allocation of the load (MW) among the various units of generating stations in such a way that, the overall cost of generation for the given load demand is minimum. This is an optimization problem which needs to be resolved as quick as possible. For a given load demand, power flow study can be used to calculate active and reactive power generations, line flows and losses. The study also furnishes some control parameters such as the magnitude of voltage and voltage phase differences. The economic load dispatch problem is the results of various power flow studies, where a particular power flow study result is considered more appropriate in terms of cost of generation. The solution to this problem cannot be optimal unless otherwise all system constraints are met. The problem of economic operation of the power system involves two sub-problems, namely, unit commitment (UC) and economic dispatch (ED). While unit commitment (UC) is an off-line problem, economic dispatch (ED) is an area of online concern. The commitment decisions are made many weeks or months in advance. The decision to commit a generating unit to be able to produce electricity means that the power utility is willing to incur fixed costs related to unit startup in order to have that generating units ready and available to generate electricity in real time. Large turbine or nuclear plant generators with large start-up costs cannot run optimally if their output is determined using a singleperiod analysis (a "period" in the electric power industry usually refers to a length of time of about an hour). Instead, their operation must be scheduled over a longer period of time, usually weeks or months. A power utility would need a forecast of demand weeks in advance before turning on a generator with a long minimum run time [3]. They would need to study the demand forecast over that period of time and decide the lowest-cost mix of generation units that would meet the demand needed. While the procedure of all ocating committed generating units to satisfy customer load demands within a given operational conditions is referred to as "economic dispatch" or "optimal power flow."

Economic Load Dispatch are usually influence by factors such as high operating cost (fuel cost) and transmission losses. The Economic Load Dispatch requires the generation facilities to plan and forecast optimal energy dispatch. Hence the concept is the optimal selection of the generating units in such an economic manner that the total cost of supplying the dynamic requirements of the system is minimized [4].

*Corresponding author: Osaremwinda OP, Department of Electrical and Electronics Engineering, Federal University of Technology, Minna, Nigeria, Tel: 2348023098495; E-mail: zolotrainer@yahoo.com

Received September 22, 2017; Accepted December 05, 2017; Published December 12, 2017

Citation: Osaremwinda OP, Nwohu MN, Kolo JG (2017) A Comparative Study of Meta-heuristics Algorithms in evaluation of Economic Load Dispatch Problems in Power Generating Station with Matlab Codes. J Electr Electron Syst 6: 245. doi: 10.4172/2332-0796.1000245

Copyright: () 2017 Osaremwinda OP, et al. This is an open-access article distributed under the terms of the Creative Commons Attribution License, which permits unrestricted use, distribution, and reproduction in any medium, provided the original author and source are credited. 


\section{Formulation of ELD problem}

The ELD problem is an optimization problem that determines the power output of each generating units that will amount to the lowest fuel cost.

Minimize $\mathrm{C}(\mathrm{x}, \mathrm{u})$

Subject to:

$\mathrm{G}(\mathrm{x}, \mathrm{u})=0$

$\mathrm{H}(\mathrm{x}, \mathrm{u}) \leq 0$

Where, $\mathrm{C}=$ cost function,

$\mathrm{x}=\mathrm{vector}$ of dependent variables (generating cost)

$\mathrm{u}=\mathrm{vector}$ of control variables (generator outputs)

$\mathrm{G}(\mathrm{x}, \mathrm{u})=$ set of non-linear quality constraints (power balance)

$\mathrm{H}(\mathrm{x}, \mathrm{u})=$ set of inequality constraints (limits in generator outputs).

The objective of the Economic Load Dispatch is to minimize the total operating cost of a power system by adjusting the power output of each of the generators connected to the grid, while satisfying the total load demand plus transmission losses within generator limits.

The generator cost curves are modeled with smooth quadratic (objective) function given by eqn. (4) subject to the equality (power balance) and inequality (generation limits) constraints in eqns. (5) and (7) respectively [6].

Minimize the objective function,

$F_{i} P_{i}=\sum_{i=1}^{n_{g}}\left(\gamma_{i} P_{i}^{2}+\beta_{i} P_{i}+\alpha_{i}\right)$

Subject to power balance equation (equality constraint)

$\sum_{i=1}^{n_{g}} P_{i}=P_{D}+P_{L}$

The system losses can be determined by means of a power flow equation solution which is expressed in krons loss formula in eqn. (6)

$P_{L}=\sum_{i=1}^{n_{g}} \sum_{j=1}^{n_{g}} P_{i} B_{i j} P_{j}+\sum_{i=1}^{n_{g}} B_{0 i} P_{i}+B_{00}$

And the (generating limits) inequality constraints,

$$
P_{i(\min )} \leq P_{i} \leq P_{i(\max )}
$$

Where $F_{i} P_{i}$ is the total generation cost, $\alpha_{i}, \beta_{i}, \gamma_{i}$ are cost coefficients, $P_{D}$ is the load demand, $P_{i}$ is real power generation of unit i, $P_{j}$ is real power generation of unit $\mathrm{j}, P_{L}$ is power transmission loss, $n_{g}$ is number of dispatchable generating plants, $P_{i(\min )}$ and $P_{i(\max )}$ are the minimum and maximum power generation limits respectively also $B_{i j}, B_{o i}$ and $B_{o o}$ are $B$-coefficient [5].

\section{Operating cost of a thermal plant}

In economic scheduling of different generating plants, the total operating cost is minimal in a Thermal Plant [5]. This generally comprises of the input to the thermal power plant which is expressed in $\mathrm{Btu} / \mathrm{h}$ or $\mathrm{Kcal} / \mathrm{h}$ and the output in MW.

The input-output curve of a thermal unit is regarded as heat-rate curve and it is usually a graph drawn between fuel input in Btu/h or $\mathrm{kcal} / \mathrm{h}$ and power output in MW on the $\mathrm{x}$-axis and $\mathrm{y}$-axis, respectively [7-9].
A typical heat-rate curve for a thermal unit is given in Figure 1, while Figure 2 shows the ordinate of heat-rate curve from Btu/h to $\mathrm{N} / \mathrm{h}$ results in the fuel-cost curve.

Hence, the fuel cost of generator $i$ can be written as a quadratic function of real power generation as shown in eqn. (8).

$$
C_{i}=\alpha_{i}+\beta_{i} P_{i}+\lambda_{i} P_{i}^{2}
$$

Incremental Fuel-cost curve is obtained by plotting the derivative of the fuel-cost curve versus the real power as shown in Figure 3. It is a measure of how costly it will be to produce the next increment of power. It is drawn by taking the incremental fuel cost in N/MWh as an input on the $\mathrm{y}$-axis and real power in MW as output on the $\mathrm{x}$-axis.

$$
\frac{d C_{i}}{d P_{i}}=2 \gamma_{i} P_{i \mathrm{i}}+\beta_{i}
$$

The total operating cost includes the fuel cost, the labor cost and maintenance [6].

\section{Research Methodology}

Economic Load Dispatch problem was formulated using mathematical illustrations and MATLAB codes.

The ELD problem is formulated as minimizing a scalar objective function through the optimal operation of a vector of controls parameters. This is mathematically illustrated in eqns. (1-3) and diagrammatically represented in Figure 4 [9].

The approach consists of two main components: the controlling device and the controlled device are shown in Figure 4. The ELD problem is one that involves the optimal set of generating units. This minimizes the operating cost (mainly fuel cost). The controlled device is the generating cost model, while the solution algorithm (SA) is the

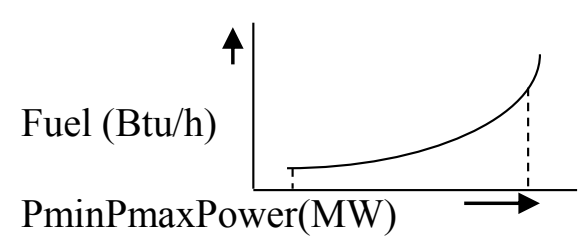

Figure 1: Heat curve of a Thermal Unit.

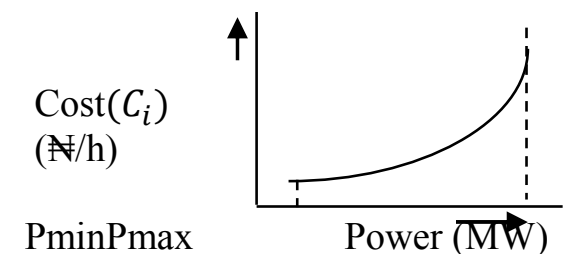

Figure 2: Fuel-cost curve characteristic of a Thermal Unit.

Incremental Fuel $\operatorname{Cost}\left(\lambda_{i}\right)$

(N/MWh)

Power (MW)

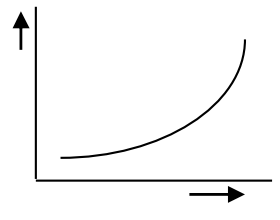

Figure 3: Incremental Fuel-Cost Curve of a Thermal Unit. 


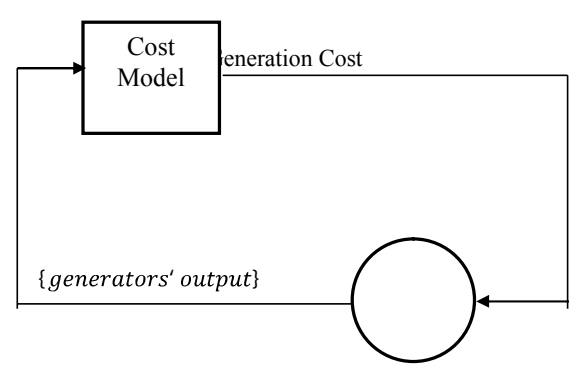

Figure 4: Diagrammatic Representation of Design Approach.

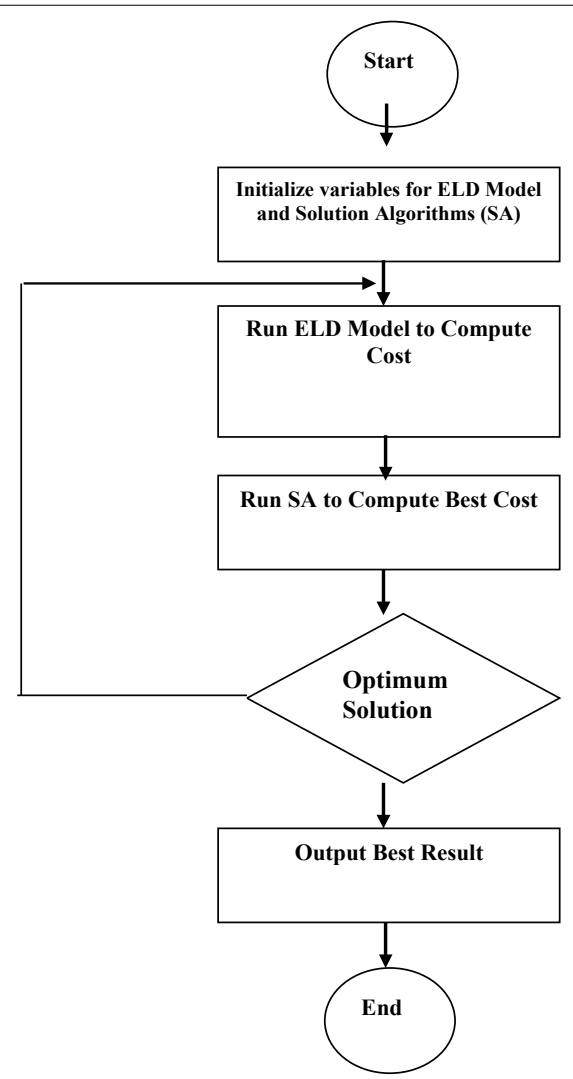

Figure 5: Flow chart of ELD Optimization using Solution Algorithms.

controlling device. A vector of generators output is inputted into the generation cost model, which produces a scalar cost of generating those power outputs. The generation cost is passed on to the solution algorithm to be reduced, an iterative process which continues until either a best cost lesser than acceptable minimum is achieved or number of maximum iterations is reached. Figure 5 show a flow diagram for ELD optimization using Solution Algorithms [9].

\section{Formulation of economic load dispatch problem}

The ELD problem is an optimization problem with an aim to find the optimal combination of committed online power generators that will reduce the total operating cost to meet the total system's power demand while satisfying equality (power balance) and inequality (generation limits) constraints. This is usually done over a period of one hour. The constraints in a practical generator include minimum and maximum generation limits, power balance, ramp rate limits and prohibited operating zones.
For a thermal generating station, the unit fuel cost is shown in the quadratic form in Figure 2, and the goal is to minimize the total operating cost as in eqns. (10) and (11), subject to the generating limit constraint of eqn. (14), power balance constraint of eqns. (12), ramprate limits constraints of eqn. (15), and prohibited operating zones constraints of eqns. (16-18).

Considering a thermal power station of $i^{\text {th }}$ generating units $G_{1}$, $G_{2}, \ldots \ldots . G_{i}$ delivering powers $P_{g 1}, P_{g 2}, \ldots \ldots, P_{g i}$ respectively connected to a transmission network as shown in Figure 6 . Where $P_{D}$ is the total power demand and $P_{L}$ is the total power losses.Each unit has its cost function $C$. The task here is to find the combination of the real power generation for all units such that the total generation $\operatorname{cost} C_{T}$ is minimized [9].

$$
\begin{aligned}
& \min C_{T}=\sum_{i=1}^{N} C_{i} \\
& =\sum_{i=1}^{N} \alpha_{i}+\beta_{i} P_{g i}+\gamma_{i} P_{g i}^{2}
\end{aligned}
$$

Subject to

$$
\begin{aligned}
& \sum_{i=1}^{N} P_{g i}=P_{D}+P_{L}(\text { for } i=1,2 \ldots . i) \\
& P_{L}=\sum_{i=1}^{N} P_{g i} B_{i j} P_{g j}
\end{aligned}
$$

Subject to

$$
P_{\text {gimin }} \leq P_{\text {gi }} \leq P_{\text {gimax }}(\text { fori }=1,2 \ldots i)
$$

Where $C_{T}$ is the total generation cost, $C i$ is the generation cost of $\mathrm{i}^{\text {th }}$ unit, $\alpha_{i}, \beta_{i}, \gamma_{i}$ are cost coefficients, $P_{D}$ is the load demand, $P_{g i}$ is real power generation of unit $i, P_{g j}$ is real power generation of unit $j, P_{L}$ is power transmission loss, $N$ is number of dispatchable generating plants, $B_{i j}$ is B-coefficient, $P_{(i(\min ))}$ and $P_{(i(\max ))}$ are the minimum and maximum power generation limits respectively.

Considering also the ramp-rate limits and prohibited zone, eqn. (14) is modified as eqn. (15),

$$
\max \left(P_{\text {imin }}, P_{i}^{o}-D R_{i}\right) \leq P_{i} \leq \min \left(P_{\text {imax }}, P_{i}^{o}+U R_{i}\right)
$$

Where $P_{i}^{o}$ is the previous output, $P_{i}$ is the present output, DR and $\mathrm{UR}$ are the down and up ramp-rate limits

The prohibited zones are described by the following inequality constraints [9].

$$
\begin{aligned}
& P_{g i}^{\min } \leq P_{g i} \leq P_{g i, 1}^{l} \\
& P_{g i, k-1}^{u} \leq P_{g i} \leq P_{g i, k}^{l}
\end{aligned}
$$

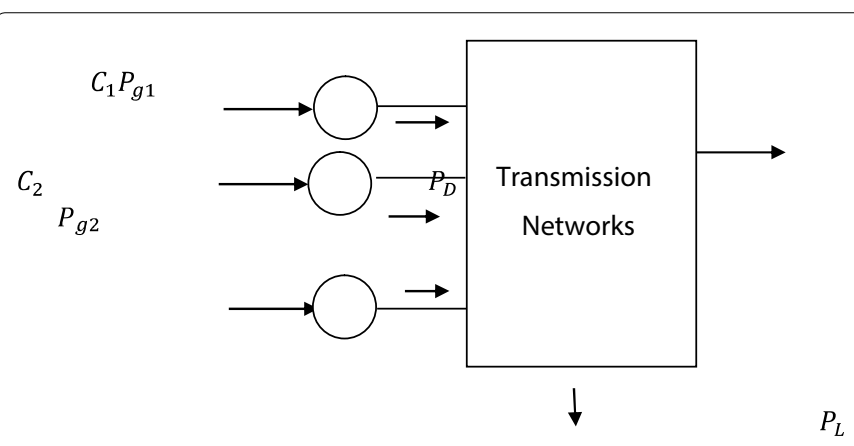

Figure 6: Generating units connected through Transmission Network. 
Citation: Osaremwinda OP, Nwohu MN, Kolo JG (2017) A Comparative Study of Meta-heuristics Algorithms in evaluation of Economic Load Dispatch Problems in Power Generating Station with Matlab Codes. J Electr Electron Syst 6: 245. doi: 10.4172/2332-0796.1000245

$$
P_{g i, z o i}^{u} \leq P_{g i} \leq P_{g i}^{\max }
$$

Where $P_{g i, k}^{l}$ and $P_{g i, k}^{u}$ are lower and upper bounds of the $k_{t h}$ prohibited zone of unit $I$ respectively, $k$ is the index of prohibited zone, $z o i$ (Tables 1 and 2).

Using the Matlab codes [7], the ELD was modeled from the concept of ELD problem defined in eqn. (10) through to eqn. (18) above showing the power demand, minimum and maximum generating limits, cost coefficients, ramp-rate limits, prohibited zone and B-coefficient. This is model as a function and called Create Model ( ) as shown Figures 7 and 8.

Applying eqns. (10-18), The Model Calculation for the Economic Load Dispatch problem was formulated and the Load apportioned to various generating units $\left(P_{\mathrm{si}}\right)$, Total generated power (PTotal), Total generation cost (CTotal) and Total power losses (PL) were calculated as shown in Figures 9 and 10.

\section{Optimization approach}

A main task in the optimization process is constraints handling. The application of the solution algorithm (SA) to solving constrained problems in the ELD involves various techniques of handling constraints, to keep the control variables in feasible region, where all the constraints are satisfied. A technique for handling these constraints constitutes the efficiency within the solution Algorithms to solving this ELD problem [9].

\section{Simulation Approach (Application of Solution Algorithms to Solve ELD)}

The modeling and program development of Solution Algorithm and the ELD problem were accomplished by using MATLAB R2008b software. All program were ran on Intel (R) $2.60 \mathrm{GHz}$ CPU, 2 GB RAM, Window 7 Computer and the modeled ELD was used to evaluate the

\begin{tabular}{|c|c|c|c|c|c|}
\hline Gen units & $\begin{array}{c}(\alpha) \\
(N / h)\end{array}$ & $\begin{array}{c}(\beta) \\
(\mathrm{N} / \mathrm{MWh})\end{array}$ & $\begin{array}{c}(\gamma) \\
\left(\mathrm{N} / \mathbf{M W}^{2} \mathrm{~h}\right)\end{array}$ & Pmin & Pmax \\
\hline 1 & 2131.1667 & 13.1 & 0.186 & 55 & 220 \\
\hline 2 & 2131.1667 & 13.1 & 0.186 & 55 & 220 \\
\hline 3 & 2131.1667 & 13.1 & 0.186 & 55 & 220 \\
\hline 4 & 2131.1667 & 13.1 & 0.186 & 55 & 220 \\
\hline 5 & 2131.1667 & 13.1 & 0.186 & 55 & 220 \\
\hline 6 & 2131.1667 & 13.1 & 0.186 & 55 & 220 \\
\hline
\end{tabular}

Table 1: Generator data of Egbin Thermal power station (six generating units) [8].

\begin{tabular}{|l|l|l|l|l|l|}
\hline B-coefficient data & \multicolumn{1}{l|}{} \\
\hline 0.00099 & 0.00016 & -0.0002 & -0.0002 & -0.0002 & -0.00015 \\
\hline 0.00014 & 0.00012 & -0.0001 & -0.0001 & 0.0001 & 0.00012 \\
\hline-0.0001 & -0.0001 & 0.00013 & 0.00013 & -0.0001 & -00012 \\
\hline-0.0001 & -0.0001 & -0.0001 & 0.00014 & 0.00013 & -0.0001 \\
\hline-0.0001 & -0.0001 & 0.00013 & 0.00013 & -0.0001 \\
\hline 0.00015 & -0.0001 & -0.0001 & -0.0001 & -0.0001 & 0.00012 \\
\hline
\end{tabular}

Table 2: Power Losses (B-coefficient) [8].

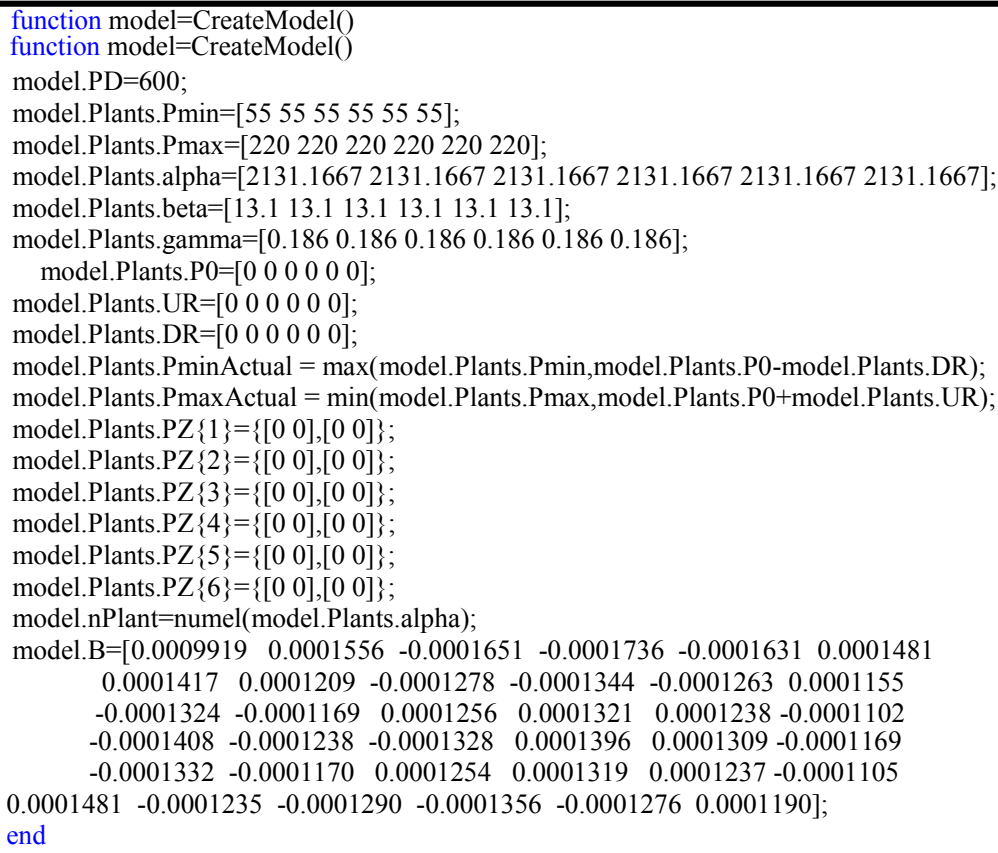

Figure 7: Create Model (Model of ELD problem in MatLab codes. 
Citation: Osaremwinda OP, Nwohu MN, Kolo JG (2017) A Comparative Study of Meta-heuristics Algorithms in evaluation of Economic Load Dispatch Problems in Power Generating Station with Matlab Codes. J Electr Electron Syst 6: 245. doi: 10.4172/2332-0796.1000245

Page 5 of 6

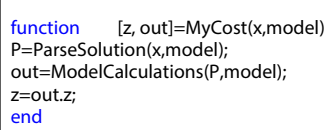

Figure 8: Cost function in ELD problem formulation in MATLAB codes.

function out $=$ ModelCalculations(P,model)

alpha=model.Plants.alpha;

beta=model.Plants.beta;

function out $=$ ModelCalculations $(\mathrm{P}$, model $)$

alpha=model.Plants.alpha;

beta=model.Plants.beta;

gamma=model.Plants.gamma;

$$
\mathrm{C}=\text { alpha+beta. } * \mathrm{P}+\text { gamma. } * \mathrm{P} . * \mathrm{P} \text {; }
$$

CTotal $=\operatorname{sum}(\mathrm{C})$;

$\mathrm{B}=$ model. $\mathrm{B}$;

$\mathrm{PL}=\mathrm{P}^{*} \mathrm{~B} * \mathrm{P}^{\prime}$

$\mathrm{PTotal}=\operatorname{sum}(\mathrm{P})$;

$\mathrm{PD}=$ model.PD;

PowerBalanceViolation $=\max (1-(\mathrm{PTotal}-\mathrm{PL}) / \mathrm{PD}, 0)$; $\mathrm{q}=100$

$\mathrm{z}=$ CTotal $*(1+\mathrm{q} *$ PowerBalanceViolation $)$;

$\mathrm{Pg}=\operatorname{sum}(\mathrm{P})$;

$P L L=P g-P D$

out. $\mathrm{P}=\mathrm{P}$;

out.PTotal $=$ PTotal;

out. $\mathrm{C}=\mathrm{C}$;

out.CTotal $=$ CTotal;

out.PLL=PLL;

$$
\text { out.PL=PL; }
$$

out.PowerBalanceViolation=PowerBalanceViolation;

out. $\mathrm{Z}=\mathrm{Z}$;

fprintf('Power Demand $\quad=\% \mathrm{f} \mathrm{MW \backslash n',PD);}$

fprintf('Total Generation $\quad=\% \mathrm{f} \mathrm{MW \backslash n',Pg)}$

fprintf('Total Losses $\quad=\% \mathrm{f} \mathrm{MW \backslash n',PLL)}$

fprintf('Total Cost of Generation = \% $\mathrm{N} / \mathrm{hr} \backslash \mathrm{n}$ ',CTotal);

Figure 9: Model Calculation of the ELD in MatLab codes.

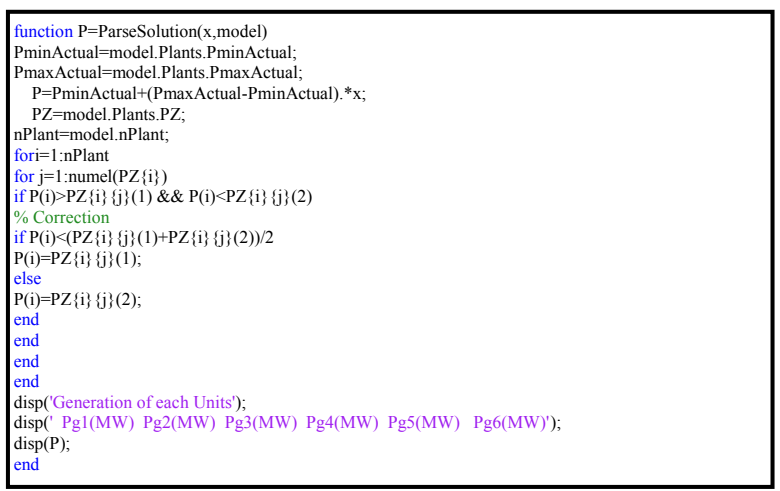

Figure 10: Parse solution of the ELD problem in MATLAB codes

effectiveness and efficiency of these algorithms (ACSA and PSO), the case study involving six generating units (Egbin thermal station) were applied.

The objective was to minimize the total operating cost, while satisfying the system constraints under the allowable limits.
The network data (Generator data \& B-coefficient) were obtained [8] which were presented in Tables 1 and 2.

The outputs results of the optimization tools which were evaluated are as follows;

- Loads apportioned to the various generating units

- Total Generated Power

- Total Power Loss

- Total Generation Cost.

\section{Application of solution algorithms in solving ELD problem in MATLAB codes}

The Create Model, Cost Function modeled in Figures 7 and 8 are

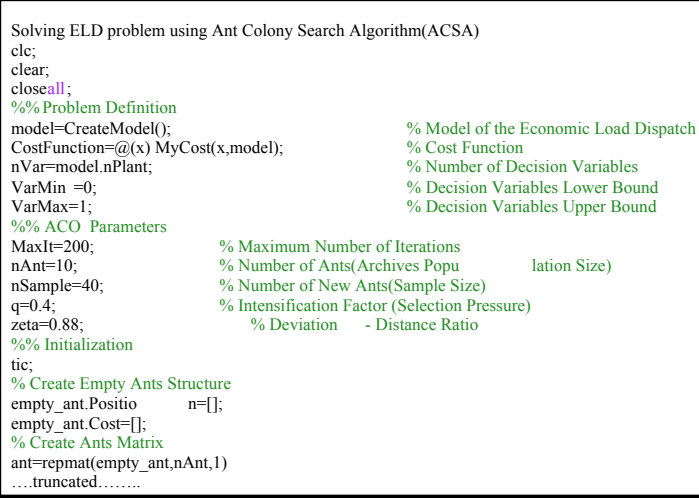

Figure 11: Solving ELD problem using ACSA in MATLAB code [7]

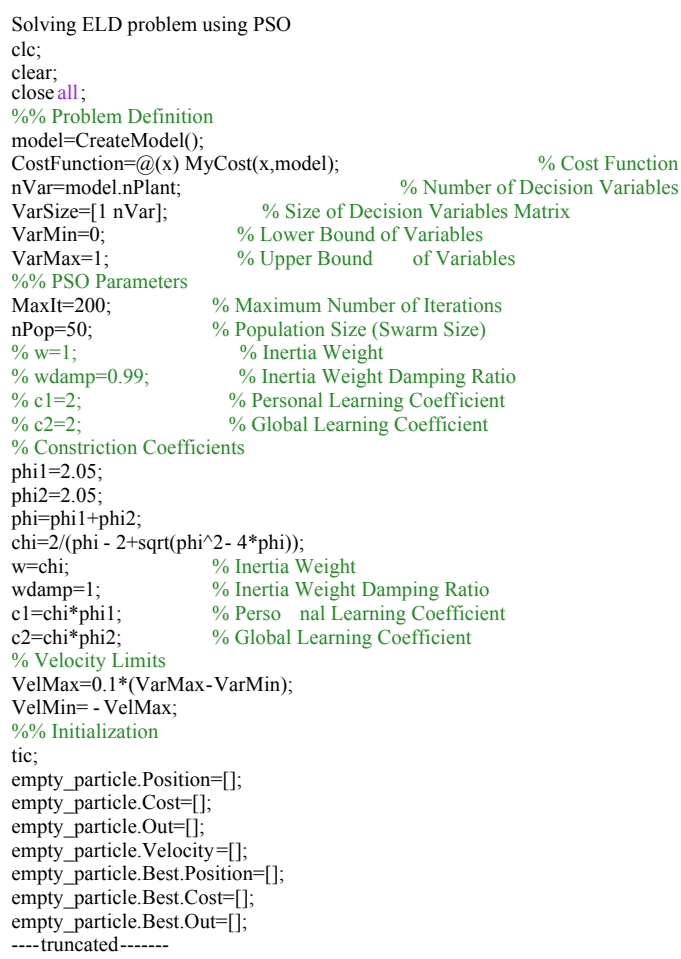

Figure 12: Solving ELD problem using PSO in MATLAB codes [7] 
Citation: Osaremwinda OP, Nwohu MN, Kolo JG (2017) A Comparative Study of Meta-heuristics Algorithms in evaluation of Economic Load Dispatch Problems in Power Generating Station with Matlab Codes. J Electr Electron Syst 6: 245. doi: 10.4172/2332-0796.1000245

Page 6 of 6

\begin{tabular}{|l|c|c|}
\hline Gen Outputs & ACSA & PSO \\
\hline G1 & 84.723 & 82.348 \\
\hline G2 & 103.562 & 103.356 \\
\hline G3 & 103.894 & 106.174 \\
\hline G4 & 108.181 & 105.734 \\
\hline G5 & 97.475 & 97.865 \\
\hline G6 & 102.176 & 104.654 \\
\hline Power Demand(MW) & 600.000 & 600.000 \\
\hline Total Power Generated(MW) & 600.011 & 600.130 \\
\hline Total Power Losses & 0.011 & 0,130 \\
\hline Total Generation Cost & 31870.672 & 31891.680 \\
\hline
\end{tabular}

Table 3: Results from simulation using Power Demand of 600MW (comparison between PSO and ACSA).

called into the solution Algorithms as shown in Figures 11 and 12 for ACSA, and PSO, respectively.

\section{Results}

Results from simulation using Power Demand of 600 MW (comparison between PSO and ACSA) is shown in Table 3.

\section{Discussion of Results}

Table 3 shows that the ACSA has effectively reduced the operating cost by $0.07 \%$ as compared with that of PSO.

\section{Conclusion}

The ELD problem has been successfully modeled and the Solution Algorithm applied to the modeled ELD problem in power generating

station in this paper. Subsequently, results shows that ACSA has effectively minimized the operating cost as compared to that of PSO.

\section{References}

1. Thakral DR, Rai HM (2008) Ant Colony Optimization: A new approach for economic load dispatch. International Journal of Research in Engineering and Applied Sciences 2: 59-69.

2. Faheemullah S, Pervez H, Mirani M, Aslam U (2012) Multi Criteria Optimization Algorithm for Economic Dispatch Complications for Sustainable Interconnected Power System. International Journal of Computer Applications 50: 22-25.

3. Dulton JA (2017) Economic Dispatch and Operations of Electric Utilities Energy Markets, Policy, and Regulation.

4. Karakonstantis I, Vlachos A (2015) Ant Colony Optimization for Continuous Domains applied to Emission and Economic Dispatch Problems. Journal of Information and Optimization Sciences 36: 23-42.

5. Girish K, Rameshawar S (2014) Economic Dispatch of Power system optimization with power generation schedule using evolutionary techniques. Internal Journal of Advanced Research in Electrical, Electronics and Instrumentation Engineering, pp: 10715-10722.

6. Swarup KS (2005) Ant colony optimization for economic generator scheduling and load dispatch. Proceedings of the $6^{\text {th }}$ WSEAS Int Conf on Evolutionary Computing, Lisbon, pp: 167-175.

7. Mostapha KHS (2015) Ant Colony Optimization for continuous domain (MATLAB programme).

8. Nwohu MN, Osaremwinda OP (2017) Evaluation of Economic Load Dispatch in power generating stations by use of Ant Colony Search Algorithms. International Journal of Research Studies in Electrical and Electronics Engineering 3: 20-29.

9. Orike S (2015) Investigating Evolutary Computation with Smart Mutation for three types of Economic Load Dispatch optimization problem. Heriot-watt university, Edinburgh UK. 